Planetary Systems in the Universe - Observation, Formation and Evolution

Proceedings IAU Symposium No. 202, (c)2004 IAU

Alan Penny, Pawel Artymowicz, Anne-Marie Lagrange, \& Sara Russell, eds.

\title{
Gas evolution in protoplanetary disks
}

W.F. Thi, E.F. van Dishoeck, G.J. van Zadelhoff

Leiden Observatory, P.O. Box 9513, 2300 Leiden, The Netherlands.

G.A. Blake, V. Mannings, A.I. Sargent

California Institute of Technology, Pasadena, CA, USA.

J. Horn, E.E. Becklin

UCLA, Los Angeles, CA, USA.

M.E. van den Ancker

Harvard-Smithsonian Center for Astrophysics, Cambridge, MA, USA.

A. Natta

Osservatorio Astrofisico di Arcetri, Firenze, Italy.

\begin{abstract}
We present the results of a study of the evolution of the gas and dust in disks around T Tauri, Herbig Ae and Vega-like stars. We observed the two lowest rotational lines of $\mathrm{H}_{2}$ with the ISO-SWS as well as ${ }^{12} \mathrm{CO} 3-2$ and ${ }^{13} \mathrm{CO} 3-2$ with the JCMT, and CO 6-5 with the CSO. The $\mathrm{H}_{2}$ lines trace the warm $(\sim 100 \mathrm{~K})$ inner region whereas the CO lines probe the colder outer disks. Substantial amounts of $\mathrm{H}_{2}$ have been detected toward T Tauri and Herbig Ae stars, and, surprisingly, also around three Vega-like objects (49 Cet, HD 135344 and $\beta$ Pictoris). In contrast with previous conclusions derived from $\mathrm{CO}$ data, a significant mass of warm gas is found to persist up to ages of several tens of Myrs, suggesting that slow formation of gas-rich giant planets is possible.
\end{abstract}

\section{Introduction}

It is well recognized that many low $\left(<1 \mathrm{M}_{\odot}\right)$ and intermediate mass $\left(1-5 \mathrm{M}_{\odot}\right)$ pre-main sequence stars are surrounded by $10^{-3}-10^{-1} \mathrm{M}_{\odot}$ of gas and dust (Mundy et al. 2000). Surveys with millimeter interferometers reveal that the matter is in the form of a disk of radius 100-400 AU in Keplerian rotation (e.g. Dutrey et al. 1996, Mannings \& Sargent 2000). The disks contain enough material for the formation of giant planets. Their main constituent - by a factor of 100 - is molecular hydrogen $\left(\mathrm{H}_{2}\right)$ which can be incorporated in the atmospheres of giant gaseous planets. Direct observation of $\mathrm{H}_{2}$ is therefore of prime importance, but Earth-based searches are hampered by the severe telluric absorption in the mid-infrared. The Short Wavelength Spectrometer (SWS) on board the Infrared Space Observatory (ISO) has allowed us to perform the first study of $\mathrm{H}_{2}$ emission from $15 \mathrm{~T}$ Tauri and Herbig Ae stars with disks. In addition, three 
older objects - 49 Cet, HD 135344 and $\beta$ Pictoris - have been observed, so that our objects cover the $(1-20) \times 10^{6}$ yr age range.

Current estimates of the disk gas mass rely on $\mathrm{CO}$ observations which require a conversion factor between the $\mathrm{CO}$ flux and $\mathrm{H}_{2}$ mass. This conversion factor varies from source to source, depending on spectral type of the central star and the density and temperature structure of the disks. Processes such as photodissociation and freeze-out onto grain surfaces have been invoked to explain the CO "depletion" (Kamp \& Bertoldi 2000). Direct observations of the lowest pure-rotational $\mathrm{H}_{2}$ lines at $17 \mu \mathrm{m}(J=3-1)$ and $28 \mu \mathrm{m}(J=2-0)$ probe the (inner) warm region (100-200 K) of disks, the most likely area for the formation of planets. Observations of high $-J$ CO transitions with the JCMT and CSO extend the study to the colder $(<80 \mathrm{~K})$ part of disks. Together with literature data on the millimeter continuum, both the warm and cold gas and dust are probed.

An important constraint on planet formation theories is the timescale of the dissipation of disks (Lissauer 1993). In the core accretion model, the growth of giant planets is slow and limited by the lifetime of the gas, whereas in the disk instability model planets can be built in a very short time provided the disk has a large mass initially. Our observations provide constraints on the evolution of the gas. A short-lived gas disk will also prohibit migration of giant planets triggered by interactions with the disk. Detailed reports can be found in Thi et al. $(1999,2000 a, b)$.

\section{Observation and Results}

The observations carried out with the ISO-SWS suffer strongly from cosmic ray bombardments. The use of dedicated data reduction software which takes advantage of multiple redundancies in the observational procedure allows us to recover the initial sensitivity (Valentijn \& Thi 2000). Figure 1 shows examples of obtained spectra. $\mathrm{H}_{2}$ lines are detected toward many $\mathrm{T}$ Tauri and Herbig Ae stars, and, surprisingly, also toward the older objects. The higher $\mathrm{S}(3)$ transition is not detected, limiting the amount of hot $(T>200 \mathrm{~K})$ gas. The $\mathrm{H}_{2}$ lines are optically thin, and their ratio gives directly the kinetic temperature assuming LTE. The inferred warm $(100-220 \mathrm{~K})$ gas masses are $(0.1-10) 10^{-3} \mathrm{M}_{\odot}$, corresponding to $1-10 \%$ of the mass derived from the dust $1.3 \mathrm{~mm}$ continuum assuming a gas/dust ratio of 100 for the pre-main sequence stars. There is no apparent trend with spectral type. For the Vega-type stars, the fraction can increase to $100 \%$. The CO spectra (cf. Figure 1) exhibit the "double-peak" profile consistent with emission from a disk in Keplerian rotation. Assuming $\mathrm{H}_{2} / \mathrm{CO}=10^{4}$ and a gas/dust ratio of 100 , the derived "cold" gas masses from ${ }^{13} \mathrm{CO}$ are factors $10-200$ lower than the masses deduced from the $1.3 \mathrm{~mm}$ dust emission.

\section{Discussion}

The "warm" $\mathrm{H}_{2}$ gas appears to constitute the major gaseous component of Vegalike objects, whereas in young objects the "cold" component dominates the gas mass. This cold gas is not well traced by CO, which is substantially depleted 

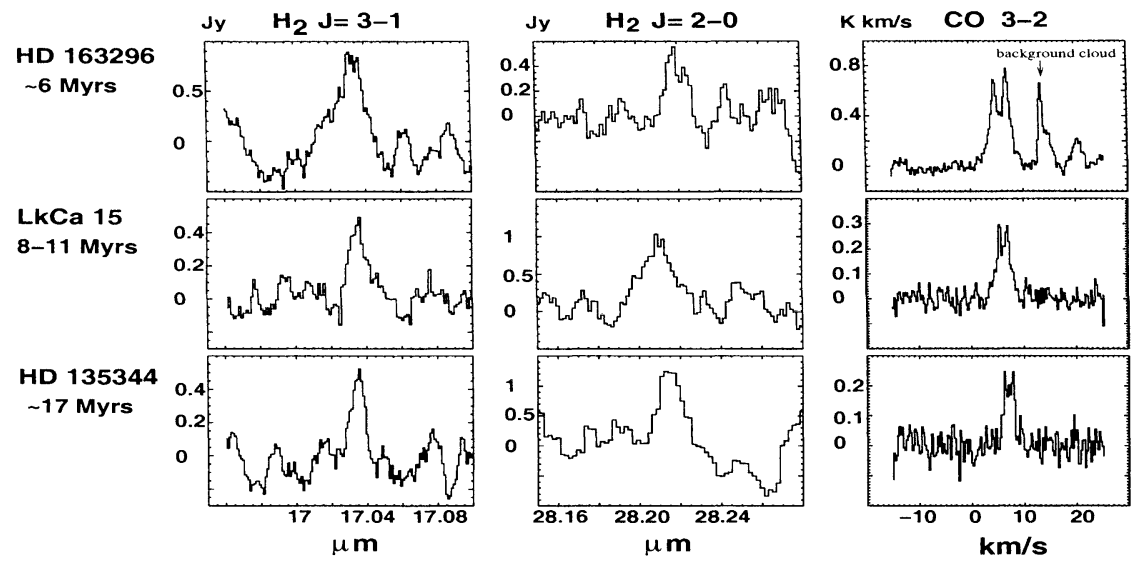

Figure 1. $\mathrm{H}_{2} \mathrm{~S}(1), \mathrm{S}(0)$ and ${ }^{12} \mathrm{CO} 3-2$ spectra of three selected objects in our sample. The ages of the stars are estimated using pre-main-sequence evolutionary tracks from Siess, Forestini, \& Bertout (2000).

due to photodissociation and freeze-out. The amount of warm gas in pre-main sequence objects is larger than can be explained by current models and more detailed calculations of the heating and cooling processes of the gas and dust need to be considered. No significant evolution of the gas and dust masses is found up to $10 \mathrm{Myrs}$, but some tendency toward decrease at later times is apparent.

\section{References}

Dutrey, A., Guilloteau, S., Duvert, G. et al. 1996, A\&A, 309, 493

Kamp, I., \& Bertoldi, F. 2000, A\&A, 353, 276

Lissauer, J. 1993, ARA\&A, 31, 129

Mannings, V., \& Sargent, A. I. 2000, ApJ, 529, 391

Mundy, L., Welch, J., \& Looney, L. 2000, in Protostars and Planets IV, ed. V. Mannings, A.P. Boss \& S.S. Russell (Tucson: Univ. Arizona Press), 355

Siess, L., Forestini, M., \& Bertout, C. 2000, A\&A, 358, 593

Thi, W.F., van Dishoeck, E.F., Blake, G.A. et al. 1999a, ApJ, 521, L63

Thi, W.F., van Dishoeck, E.F., Blake, G.A. et al. 2000a, ApJ, submitted

Thi, W.F., Blake, G.A., van Dishoeck, E.F. et al. 2000b, Nature, in press

Valentijn, E. A., \& Thi, W. F. 2000, Experimental Astronomy 10, 215 